\title{
Systems Medicine and Infection
}

\author{
Ruth Bowness \\ School of Medicine, University of St Andrews, \\ North Haugh, St Andrews, United Kingdom
}

\begin{abstract}
Summary
By using a systems based approach, mathematical and computational techniques can be used to develop models that describe the important mechanisms involved in infectious diseases. An iterative approach to model development allows new discoveries to continually improve the model, and ultimately increase the accuracy of predictions.
\end{abstract}

SIR models are used to describe epidemics, predicting the extent and spread of disease.

Genome-wide genotyping and sequencing technologies can be used to identify the biological mechanisms behind diseases. These tools help to build strategies for disease prevention and treatment, an example being the recent outbreak of Ebola in West Africa where these techniques were deployed.

HIV is a complex disease where much is still to be learnt about the virus and the best effective treatment. With basic mathematical modelling techniques, significant discoveries have been made over the last 20 years. With recent technological advances, the computational resources now available and interdisciplinary cooperation, further breakthroughs are inevitable.

In TB, modelling has traditionally been empirical in nature, with clinical data providing the fuel for this top-down approach. Recently, projects have begun to use data derived from laboratory experiments and clinical trials to create mathematical models that describe the mechanisms responsible for the disease. 
A systems medicine approach to infection modelling helps identify important biological questions that then direct future experiments, the results of which improve the model in an iterative cycle. This means that data from several model systems can be integrated and synthesised to explore complex biological systems.

\section{Key words}

Infection, Mathematical, Modelling, Epidemic, Tuberculosis, HIV,

\section{Introduction}

Infectious diseases continue to be major worldwide health concerns: hepatitis $\mathrm{C}$, malaria, the human immunodeficiency virus (HIV) and tuberculosis (TB) are ongoing pandemics. A third of the world population is currently infected with the TB bacillus; and even though therapeutic drugs have slowed the threat of HIV, there is no definitive cure or viable vaccine in sight. A new way of approaching the problem is needed; this can be achieved with a systems approach. By utilising expertise across disciplines, fresh perspective and new insights can be gained.

Current modelling efforts span multiple levels in the disease system; population dynamics where the focus is on disease transmission, the progression from population level to individual level where heterogeneity is included, moving to the pathogen-host interactions ranging from molecular to cellular, to whole organism levels. Both biological experiments and mathematical modelling have been successful at elucidating the properties of a disease at a particular level, but a full understanding requires the integration of all scales. This remains a 
major challenge for systems medicine. Infectious diseases reflect an equilibrium between the host and the pathogen that is established and maintained by a broad network of interactions that occur on such scales. Maintenance and evolution of these interactions over a prolonged time frame adds further complexity to persistent infections. The understanding of a biological system requires the integration of data that are used to construct predictive models of the dynamic interactions between biological components of the complex pathogen-host system.

Mathematical and computational techniques, together with available in vitro and in vivo experimental results can be used to generate realistic pathogen-host interaction models. Often these models involve iterative rounds of development, with testing and refinement using, for example, Bayesian methods. Models created in such a manner can be used to create testable hypotheses, with much learnt about the biological system in the process (see Figure 1). Ultimately, predictions can be made that can guide clinical practice. This iterative cycle is key to the systems approach and requires efficient integration of data with firm collaboration across disciplines. The technologies and computational resources now exist to realise this systems approach, we must take advantage of the potential these provide.

In this chapter we review some basic epidemic modelling (section 2) before using two infectious disease case studies, HIV (section 3) and tuberculosis (section 4), to demonstrate how a systems approach, using simple mathematical techniques, can be used to further biological understanding and ultimately lead to changes in clinical practice. The focus in these sections is at the cellular level, describing viral/bacterial load dynamics. 


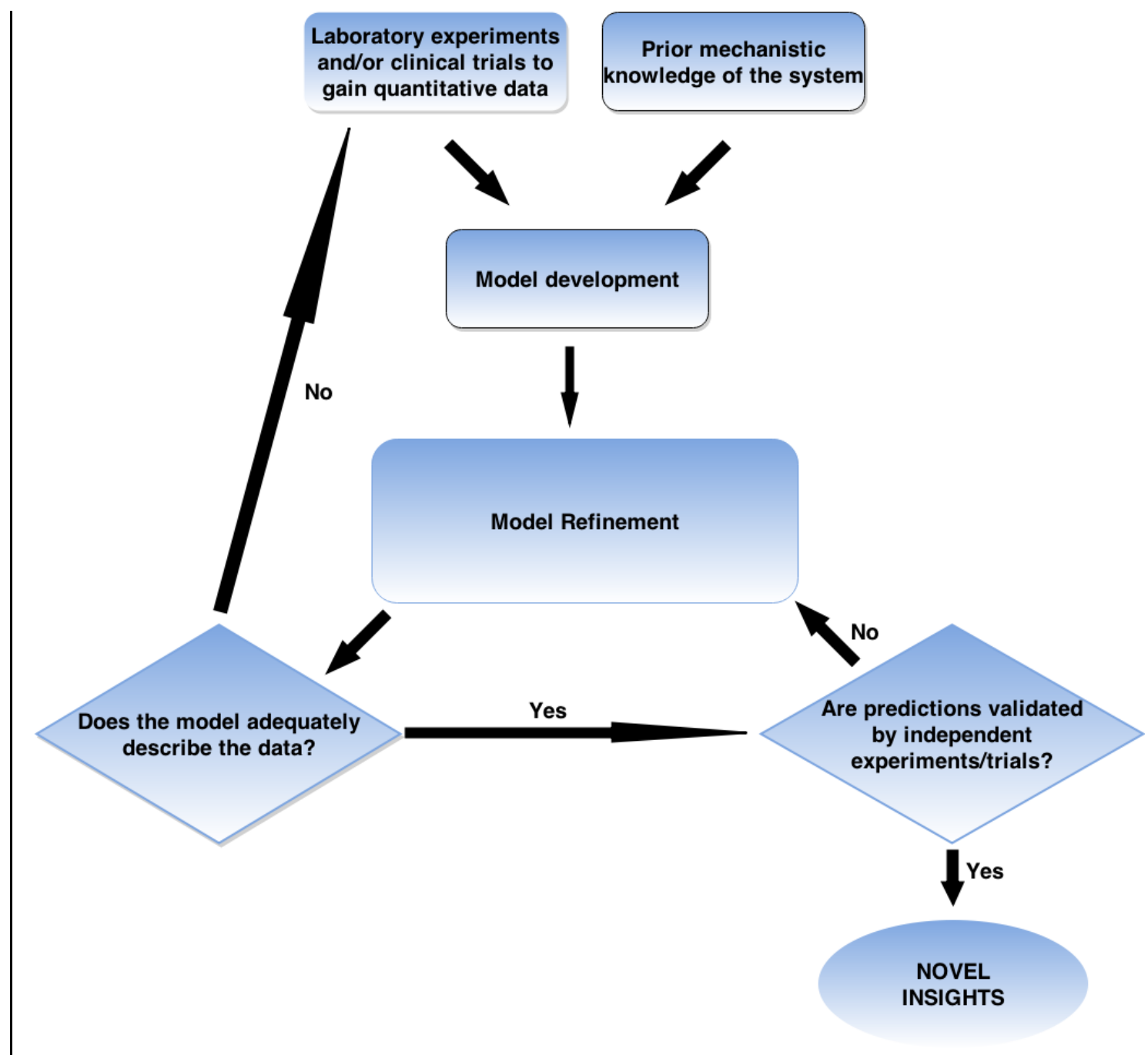




\section{Epidemic modelling}

One motivating reason for modelling the spread of infectious diseases is to understand how future outbreaks can be prevented. This can be achieved in several different ways, such as isolation or imposed travel restrictions. These measures aim at reducing contact rates, i.e. to reduce the reproduction rate of the pathogen. The effect of these depends on the particular disease and the community under consideration. Vaccination is an alternative preventive measure, which reduces the pool of susceptible individuals by imparting immunity.

In classical mathematical epidemic models [1], the total population number is assumed to be constant. A small group of infected people is introduced into a large population and a model is used to describe the spread of infection within that population as a function of time. The model consists of three subpopulations of individuals:

$\mathrm{S}(\mathrm{t})$ which denotes the number of people susceptible to the disease,

$\mathrm{I}(\mathrm{t})$ which denotes the number of people who are infected and can transmit the disease and $R(t)$ which denotes the number of removed people, i.e. those who were infected but are now recovered, are immune or have been isolated until they are recovered.

Such models are known as SIR models, where transfer between populations is restricted to $S \rightarrow I$ and $I \rightarrow R$.

Some diseases include a class in which the disease is latent, E. Such models are known as SEIR models.

Assumptions of the SIR model:

- The gain in the infective class is at a rate that is proportional to the number of infectious and susceptible people, rSI where $r>0$ is a constant parameter. 
- The susceptible population is lost at the same rate. The transfer of infected individuals to the removed class is proportional to the number of people who are infected, aI where a $>0$ is a constant. $1 / \mathrm{a}$ is a measure of the time spent in the infectious state.

- The incubation period is short enough to be negligible hence a susceptible person who catches the disease is infected immediately.

- Every person has equal probability of coming into contact with one another.

The model mechanism [2] is therefore,

$$
\begin{gathered}
\frac{d s}{d t}=-r S I, \\
\frac{d I}{d t}=r S I-a I, \\
\frac{d R}{d t}=a I,
\end{gathered}
$$

where $r>0$ is the infection rate and $a>0$ is the removal rate of infectious individuals.

Initial conditions are defined as $\mathrm{S}(0)=\mathrm{S}_{0}>0, \mathrm{I}(0)=\mathrm{I}_{0}>0$ and $\mathrm{R}(0)=0$ and the constant total population is built into the system via $\frac{d S}{d t}+\frac{d I}{d t}+\frac{d R}{d t}=0$ which implies that $S+I+R=N$, where $\mathrm{N}$ is the total population.

With this model, questions can be answered such as whether an infection will spread or not. If it does, how does it develop with time and critically, when will it start to decline? Given r, $\mathrm{a}, \mathrm{S}_{0}$ and $\mathrm{I}_{0}$ these questions can be addressed.

This type of stochastic modelling looks at the properties of an epidemic by studying a given model and its parameters. It can be shown that the most important parameter in these SIR models is the basic reproduction rate of the disease, $R_{0}=\frac{r S_{0}}{a}$, where it can be shown that when $\mathrm{R}_{0}>1$, an epidemic ensues. Another important quantity when trying to avoid an 
outbreak is the critical vaccination coverage, which is defined as $v_{C}=1-\frac{1}{R_{0}}$ if $\mathrm{R}_{0}>1$, otherwise $v_{C}=0$.

Even when trying to include as many realistic features in a model as possible there is a limit to how close a model can get to reality, and models can never completely predict what will happen in a given situation. It is, for example, nearly impossible to predict how people will adapt and change behaviour as a disease starts spreading. Having said this, models can still be very useful as guidance for health professionals when deciding about preventive measures aiming at reducing the spread of a disease. Much has been written describing mathematical models for infectious disease spread [3], [4], including stochastic epidemic models [5], [6], [7].

From traditional mathematical modelling it is clear that understanding and incorporating information from multiple scales can dramatically increase the power of such approaches. Thus, for example, emerging genome-wide genotyping and sequencing technologies are used to identify the biological mechanisms underlying the development of complex diseases and traits among populations. This allows models to build in host and pathogen features, but also points to the inclusion of larger, societal or population data. Such approaches have started to be deployed, for example in the recent epidemic outbreaks of Ebola in West Africa. Collectively, these approaches can help inform strategies for disease prevention and treatment. 


\section{HIV modelling}

In 2013, 1.5 million people died from HIV-related causes globally, having claimed an overall 39 million lives so far (World Health Organization, 2014). There is no cure for HIV infection but antiretroviral treatment (ART) can be used to control the disease. There is much still to be learnt about the virus and how best to administer effective treatment. Mathematical models developed over the last 20 years have helped to further understanding and provide new insights into the disease.

One of the simplest mathematical models to describe HIV was proposed in 1995, where a simple linear first-order equation is used to describe viral load over time [8],

$$
\frac{d V}{d t}=P-c V
$$

where $P$ represents the viral production rate and $c$ is the viral clearance rate. Immune cells, fluid flow and absorption into other cells are combined to give the overall clearance of viral peptides, $c$.

After introduction of the protease inhibitor it is assumed that the drug is completely effective, so the drug will block all viral production after being introduced. Under this assumption, $P=0$ which leaves the simple equation

$$
\frac{d V}{d t}=-c V \Rightarrow V(t)=V_{0} e^{-c t}
$$

where $V_{0}$ is the mean viral concentration in the plasma before treatment.

Using linear regression to examine the relationship between $\ln V$ against $t$ gives an estimate for $c$ and hence for the half-life of the virus in the plasma, $t_{\frac{1}{2}}=\ln \frac{2}{c}$.

If an assumption is then made that the levels of viral load measured in the plasma remain

fairly constant before treatment begins, i.e. the patient is in a quasi-steady state, $\frac{d V}{d t}=0$, then by knowing $c$ and the initial virus concentration $V_{0}$, the viral production rate before therapy 
can be computed, i.e., $P=c V_{0}$. It should be noted, however, that as this calculation is based on the assumption that the drug completely blocks virus production. This assumption is unlikely to hold, and so, experiments would measure the rate of virus clearance in the face of some residual production, and the gradient of viral decline would therefore be a lower bound of the true clearance rate. Further models with added complexities have been developed from this simple framework (see [9] for a comprehensive review).

By using relatively simple modelling techniques and trivial mathematics, fundamental information has been gained about the underlying biological mechanisms of the disease. These developments have made a substantial impact on our thinking and understanding of HIV infection. For example, because the disease can take around 10 years to develop, many thought that the disease process would be slow and treatment could be delayed until symptoms appeared. Patients were therefore not monitored very aggressively. Modelling, coupled with appropriate experiments, has revealed that HIV is a dynamic disease encompassing various different time scales. An extremely simple model involving a single linear ordinary differential equation (described above), when applied to the interpretation of clinical data, gave the first quantitative estimate of how rapidly HIV was being produced and cleared [8], [10].

Rigorous analysis of previously conducted in vivo experiments also prompted the practice of using prolonged therapy with effective drug combinations. Calculations revealed that in an average HIV-infected person, around $10^{10}$ viral particles are produced and released into bodily fluids per day [11]. It was therefore calculated that an infected person could go through about 200 replication cycles per year, with the possibility of mutating at each replication With this new information, the rapid evolution of HIV could easily be understood. Therapy with a single drug, in which a few mutations were all that were required for resistance to arise, was concluded to be a poor strategy. This demonstrated the need for combination therapy. 
Adding complexity into the modelling by considering multiple cell populations, it was found that the virus concentration in plasma has a two-phase decline. By using data obtained from patients responding well to combination therapy, estimates of how long therapy would need to be given to clear the cells responsible for producing the observed levels of virus were calculated. This led to the practice of antiretroviral drugs being taken for at least 2 or 3 years after the virus is no longer detectable in the blood [9]. This modelling work also began the process of quantifying both the level and the role of latently and long-lived infected cell populations in HIV infection.

These important breakthroughs in the understanding of the HIV and its treatment have arisen from relatively trivial mathematical modelling exercises. This effectively demonstrates how systems based, interdisciplinary approaches can make huge advances in medical treatment. 


\section{Tuberculosis modelling}

Tuberculosis remains one of the leading causes of death by infectious disease, second only to HIV/AIDS. It is estimated that one third of the world population is latently infected by Mycobacterium tuberculosis, with 2012 seeing 8.6 million people falling ill and 1.3 million dying from the disease (World Health Organization, 2014).

Although well-administered short-course chemotherapy is clinically effective [12], there are several concerns surrounding current TB treatment. The emergence of multi-drug and extensive drug resistance is a major burden since it could lead to an increase of tuberculosis cases that are hard or impossible to treat [13]. Another major issue in tuberculosis treatment is its duration, which is currently a minimum of 6 months. Shortening the duration of effective TB therapy would mean better patient compliance and lower rates of relapse and drug resistance.

Traditional approaches in tuberculosis research are based on pre-clinical experiments, in vitro and in vivo. These systems each have limitations and often, desired experiments are not feasible due to laboratory or ethical issues. Mathematical modelling alongside these assays allows hypotheses to be developed and tested, and hence further understanding of the disease by suggesting innovative approaches.

In the last 20 years, mathematical models have provided major insights in the knowledge of tuberculosis pathogenesis [14], [15], [16]. During this time, progress has also been made in the quantitative description of both pharmacokinetics (PK) and in vitro pharmacodynamics (PD) of antituberculosis drugs [17], [18], [19]. Mathematical models that arise from a systems approach offer a unique potential to establish quantitative links across multiple biological scales. Different mathematical systems capture biological complexity best at individual scales. Once mathematical models adequately describe these biological complexities on an 
individual scale, integrating the scales will then be vital in order to understand the overall dynamics of this infectious disease. In this chapter we focus on modelling at the cellular level, modelling bacterial load detected in clinical sputum samples.

It has long been noted in tuberculosis patients that decline in bacterial numbers appears to have two phases of decline. Many studies have employed non-linear mixed effects modelling techniques to fit bi-exponential functions to clinical trial data [20], [21]. For example, in [21], a bi-exponential model of the form

$$
\log _{10} C F U=\log _{10}\left(e^{\theta_{1}} e^{- \text {daye } e^{\theta_{2}}}+e^{\theta_{3}} e^{- \text {daye } e^{\theta_{4}}}\right)
$$

was used, where a $\log _{10}$ transformation of the response and a variance function were used to account for heteroscedasticity and an exponentiated parameterisation was used to enforce positivity of the parameters. These authors showed that the bi-exponential models provide a significantly better fit than a mono-exponential model. As it is known from in vitro studies that Mycobacterium tuberculosis bacteria exist in more than one cell state, the interpretation of the two-phase decline is that two subpopulations of Mycobacterium tuberculosis are present in the sputum, each declining at different rates. This has led to hypotheses about "dormant" cells being responsible for latent disease, and relapse. Consequently huge effort has gone into researching this less metabolically active subpopulation of cells.

Traditional statistical modelling techniques have also been useful for identifying trends in clinical data sets. As tuberculosis is a slow growing organism (with a generation time between 17.5-56 hours [22], [23]), it can take time to obtain microbiological results to assess the progress of treatment. For this reason, modelling techniques have been used to identify biomarkers of success. Thus the 8 -week biomarker of culture conversion was created as the most used indicator of treatment outcome [24]. More recent modelling efforts have been employed to investigate the reliability of using baseline bacterial load as an indicator for later relapse [25]. 
Although statistical modelling techniques are very powerful, fitting to the clinical data empirically in order to provide future predictions, the differential equations describing the system are employed without reference to the mechanisms underlying the biological system and so little can be learnt about the basic biology using these methods.

In contrast to statistical modelling, mathematical or mechanistic models can summarise current knowledge, and in their development, a greater understanding of the biological system can be gained. They can be used to highlight gaps in current knowledge and identify tractable biological questions. The mechanistic approach also allows us to predict how a system will shift when underlying processes change. If mechanistic models are correctly specified, they should provide better simulation and prediction properties than many current empirical models.

In TB, mechanistic models use available clinical and preclinical data to pre-define parameter values before solving the set of differential equations describing the biological system. Although there is often uncertainty when assigning parameter values, common parameter estimation techniques such as profile likelihood can be used to analyse parameter sensitivity in the system. A huge advantage of mechanistic modelling is that we are able to analyse the effect that parameters have on the bacterial load. For example, by altering killing parameters, the effect of new regimens on time to culture conversion can be analysed. Drugs targeting bacteria in different cell states can also be investigated. These simulations provide a surrogate for experiments that would not be feasible in vivo or in vitro.

This systems medicine approach means that during development of mechanistic models, important biological questions can be identified and therefore direct future experiments, the results of which will feed back into the model (see Figure 1). Hence data from several model systems (animal, human, bacterial, and computational) can be integrated and synthesised to explore the complex biological system and address relevant questions. 


\section{TB-HIV co-infection}

For tuberculosis patients co-infected with the HIV, the disease becomes more complex to treat. Most TB-HIV models address the epidemiology of co-infection. In 1992, the first model was published to quantify the consequences of the emerging TB-HIV epidemic [26]. In 2003, it was found that Antiretroviral therapy (ART) must be started early if expansion to the access of the therapy was to result in the increased control of TB [27]. Isoniazid preventative therapy (IPT) has also been modelled [28], [29], resulting in predictions of $34 \%-100 \%$ of reduction of risk of TB. Models have also been used to explore enhancements to DOTS-based programmes [30], and also to inform policy on implementation of new interventions and tools [31]. In a recent article, [32] suggest a modelling TB-HIV research agenda, based on expert discussions at a meeting convened by the TB Modelling and Analysis Consortium. Here they identified high-priority areas for future modelling efforts; the difficult diagnosis and high mortality of TB-HIV, the high risk of disease progression, TB health systems in high HIV prevalence settings, uncertainty in the natural progression of TB-HIV and combined interventions for TB-HIV. The realisation of these aims relies on a systems based approach, where modellers' and key stakeholders' collaborative efforts result in tangible progress.

Models that address the pathology of TB-HIV infected patients are also presented in the literature. Some studies attempt to understand how Mycobacterium tuberculosis affects the dynamic interaction of HIV-1 and the immune system [33], ,34]. More modelling in this area is needed if we are to understand the complex interactions between these two pathogens, and hence start to provide recommendations for improvement in treatment plans. 


\section{Conclusions}

Infectious diseases such as HIV and tuberculosis are major global health concerns and much is still unknown about the diseases. In order to aid improvements in treatment, mathematical and computational models are needed to provide a new insight.

Interdisciplinary research is often criticised as researchers from other specialties are ignorant of many fundamental concepts in the discipline they are new to. However, this can also bring advantages. In model development, there is a danger that existing beliefs can inappropriately influence judgements about model assumptions and results. When mathematicians and computer scientists are used to develop models, however, they are not influenced by current dogmas in the field and can provide a fresh perspective on the problem.

Mathematics provides a precise quantitative language to describe the relation between variables and changes in states, and in medicine we can represent mathematically the clinical course of disease, the distribution of disease across populations and over time, and the mechanisms that generate disease. We have seen in this chapter that relatively simple mathematics has led to huge leaps forward in the understanding of both HIV and tuberculosis infection. With more sophisticated analysis and ever increasing computational power, the possibility of significant breakthroughs using this systems-based approach to medical research presents an exciting prospect. 


\section{References}

1. Murray JD (2003) Mathematical Biology I: Introduction.

2. Kermack WO, McKendrick A G. 1927 Contributions to the mathematical theory of epidemics.

3. Anderson RM, May RM (1991) Infectious disease of humans. Dynamics and control

4. Diekmann O, Heesterbeek J Mathematical epidemiology of infectious diseases: model building, analysis and interpretation. 2000, Chichester: John Wiley pp. 303.

5. Britton T (2010) Stochastic epidemic models: A survey. Mathematical Biosciences 225:24-35. doi: 10.1016/j.mbs.2010.01.006

6. Bailey NT (1987) The Mathematical Theory of Infectious Diseases. Macmillan Publishing Company

7. N. G. Becker, Analysis of Infectious Disease Data, Monographs on Statistics and Applied Probability, Chapman \& Hall, London, UK, 1989

8. Ho DD, Neumann AU, Perelson AS, Chen W (1995) Rapid turnover of plasma virions and CD4 lymphocytes in HIV-1 infection. Nature 1995 Jan 12;373(6510):123-6

9. Perelson AS, Nelson PW (1999) Mathematical Analysis of HIV-1 Dynamics in Vivo. SIAM Rev 41:3-44. doi: 10.1137/S0036144598335107

10. Wei X, Ghosh SK, Taylor ME, et al. (1995) Viral dynamics in human immunodeficiency virus type 1 infection. Nature_1995 Jan 12;373(6510):117-22

11. Perelson AS, Essunger P, Cao Y, et al. (1997) Decay characteristics of HIV-1-infected compartments during combination therapy. Nature 387:188-191. doi: 10.1038/387188a0

12. Mitchison DA (2005) The diagnosis and therapy of tuberculosis during the past 100 years. Am J Respir Crit Care Med 171:699-706. doi: 10.1164/rccm.200411-1603OE

13. Gandhi NR, Moll A, Sturm AW, et al. (2006) Extensively drug-resistant tuberculosis as a cause of death in patients co-infected with tuberculosis and HIV in a rural area of South Africa. The Lancet 368:1575-1580. doi: 10.1016/S0140-6736(06)69573-1

14. Fang X, Wallqvist A, Reifman J (2009) A systems biology framework for modeling metabolic enzyme inhibition of Mycobacterium tuberculosis. BMC Systems Biology 3:92. doi: 10.1186/1752-0509-3-92

15. Segovia-Juarez JL, Ganguli S, Kirschner D (2004) Identifying control mechanisms of granuloma formation during $\mathrm{M}$. tuberculosis infection using an agent-based model. Journal of Theoretical Biology 231:357-376. doi: 10.1016/j.jtbi.2004.06.031

16. Wigginton JE, Kirschner D (2001) A model to predict cell-mediated immune regulatory mechanisms during human infection with Mycobacterium tuberculosis. J Immunol 166:1951-1967. doi: 10.4049/jimmunol.166.3.1951

17. Gumbo T (2010) New susceptibility breakpoints for first-line antituberculosis drugs based 
on antimicrobial pharmacokinetic/pharmacodynamic science and population pharmacokinetic variability. Antimicrob Agents Chemother 54:1484-1491. doi: 10.1128/AAC.01474-09

18. Jayaram R, Gaonkar S, Kaur P, et al. (2003) Pharmacokinetics-pharmacodynamics of rifampin in an aerosol infection model of tuberculosis. Antimicrob Agents Chemother 47:2118-2124. doi: 10.1128/AAC.47.7.2118-2124.2003

19. Jayaram R, Shandil RK, Gaonkar S, et al. (2004) Isoniazid pharmacokineticspharmacodynamics in an aerosol infection model of tuberculosis. Antimicrob Agents Chemother 48:2951-2957. doi: 10.1128/AAC.48.8.2951-2957.2004

20. Rustomjee R, Lienhardt C, Kanyok T, et al. (2008) A Phase II study of the sterilising activities of ofloxacin, gatifloxacin and moxifloxacin in pulmonary tuberculosis. Int $\mathrm{J}$ Tuberc Lung Dis 12:128-138.

21. Davies GR, Brindle R, Khoo SH, Aarons LJ (2006) Use of nonlinear mixed-effects analysis for improved precision of early pharmacodynamic measures in tuberculosis treatment. Antimicrob Agents Chemother 50:3154-3156. doi: 10.1128/AAC.00774-05

22. O'Sullivan DM, McHugh TD, Gillespie SH (2010) Mapping the fitness of Mycobacterium tuberculosis strains: a complex picture. J Med Microbiol 59:1533-1535. doi: 10.1099/jmm.0.019091-0

23. Shorten RJ, McGregor AC, Platt S, et al. (2013) When is an outbreak not an outbreak? Fit, divergent strains of Mycobacterium tuberculosis display independent evolution of drug resistance in a large London outbreak. J Antimicrob Chemother 68:543-549. doi: $10.1093 / \mathrm{jac} / \mathrm{dks} 430$

24. Aber VR, Nunn AJ (1978) Short term chemotherapy of tuberculosis. Factors affecting relapse following short term chemotherapy. ... of the International Union Against Tuberculosis

25. Perrin F, Woodward N, Phillips P (2010) Radiological cavitation, sputum mycobacterial load and treatment response in pulmonary tuberculosis. ... of Tuberculosis and ...

26. Schulzer M, Radhamani MP, Grzybowski S, et al. (1994) A mathematical model for the prediction of the impact of HIV infection on tuberculosis. Int J Epidemiol 23:400-407.

27. Williams BG, Dye C (2003) Antiretroviral drugs for tuberculosis control in the era of HIV/AIDS. Science 301:1535-1537. doi: 10.1126/science.1086845

28. Wilton P, Smith R, Coast J, Millar M (2001) Directly observed treatment for multidrugresistant tuberculosis: an economic evaluation in the United States of America and South Africa. ... of Tuberculosis and ...

29. Murray M (2002) Determinants of cluster distribution in the molecular epidemiology of tuberculosis. PNAS 99:1538-1543. doi: 10.1073/pnas.022618299

30. Corbett EL, Watt CJ, Walker N, et al. (2003) The Growing Burden of Tuberculosis: Global Trends and Interactions With the HIV Epidemic. Arch Intern Med 163:10091021. doi: 10.1001/archinte.163.9.1009

31. Baltussen R, Floyd K, Dye C (2005) Cost effectiveness analysis of strategies for tuberculosis control in developing countries. BMJ 331:1364-0. doi: 
10.1136/bmj.38645.660093.68

32. Houben RMGJ, Dowdy DW, Vassall A, et al. (2014) How can mathematical models advance tuberculosis control in high HIV prevalence settings? Int J Tuberc Lung Dis 18:509-514. doi: 10.5588/ijtld.13.0773

33. Kirschner D (1999) Dynamics of co-infection with M. Tuberculosis and HIV-1. Theor Popul Biol 55:94-109. doi: 10.1006/tpbi.1998.1382

34. Magombedze G, Garira W, Mwenje E (2006) Modelling the human immune response mechanisms to mycobacterium tuberculosis infection in the lungs. Math Biosci Eng 3:661-682. 


\section{Figures}

Figure 1: This schematic describes dynamic model development. Prior knowledge and data from laboratory results help to develop an initial model. Once constructed, this model is refined. After this, an iterative cycle begins where the model is tested against the available data until the model is deemed to adequately describe this data. Once this cycle is complete, the last iterative cycle is embarked upon where predictions are made and ultimately validated against an independent data set. 